\title{
Antimonotonicity and multistability in a fractional order memristive chaotic oscillator
}

Chao-Yang Chen ${ }^{1,2}$, Karthikeyan Rajagopal ${ }^{3}$, Ibrahim Ismael Hamarash ${ }^{4}$, Fahimeh Nazarimehr ${ }^{5, a}$, Fawaz E. Alsaadi ${ }^{6}$, and Tasawar Hayat ${ }^{7,8}$

${ }^{1}$ School of Information and Electrical Engineering, Hunan University of Science and Technology, Xiangtan 411201, P.R. China

${ }^{2}$ Center for Polymer Studies and Department of Physics, Boston University, Boston, MA 02215, USA

${ }^{3}$ Center for Nonlinear Dynamics, Defence University, Bishoftu, Ethiopia

${ }^{4}$ Department of Computer Science and Engineering, University of Kurdistan-Hewler, Erbil, Iraq

${ }^{5}$ Department of Biomedical Engineering, Amirkabir University of Technology, 424 Hafez Ave., Tehran 15875-4413, Iran

${ }^{6}$ Department of information Technology, Faculty of Computing and IT, King Abdulaziz University, Jeddah, Saudi Arabia

${ }^{7}$ Department of Mathematics, Quaid-I-Azam University 45320, Islamabad 44000, Pakistan

${ }^{8}$ NAAM Research Group, King Abdulaziz University, Jeddah, Saudi Arabia

Received 9 December 2018 / Received in Final form 5 February 2019 Published online 14 October 2019

\begin{abstract}
A memristor diode bridge chaotic circuit is proposed in this paper. The proposed oscillator has only one nonlinear element in the form of memristor. Dynamical properties of the proposed oscillator are investigated. The fractional order model of the oscillator is designed using Grünwald-Letnikov (GL) method. Bifurcation diagrams are plotted which shows that the proposed oscillator exhibits multistability. Finally, the antimonotonicity property of the fractional order oscillator is discussed in detail with two control parameters. Such property has not been explored for fractional order systems before.
\end{abstract}

\section{Introduction}

In the last three decades a lot of attention has been given to dynamical systems with elegant mathematical forms. In 1994, Sprott proposed a list of simplest 3 -dimensional chaotic systems [1]. Those systems have been the source of inspiration of many new simple chaotic systems [2-4]. Finding chaotic systems with special properties is very interesting since it can help us to understand the generation of chaotic attractors. There are many studies on designing chaotic flows with special structural properties such as systems with no equilibrium point [5-8], stable equilibria [9-12], line of equilibria [13], curve-equilibrium [14,15], surface-equilibrium [16-18], and infinite equilibrium points [19-21]. Chaotic systems with other properties such as fractional-order equations [22-24], multi-scroll attractors [25,26], megastability [27-30], and extreme multistability [31-34] are also attractive. Multistability in a

\footnotetext{
a e-mail: fahimenazarimehr@yahoo.com
} 




Fig. 1. Circuit structure of MDBO.

new 4-dimentional chaotic system has been investigated in [35]. In [31] multistable memristor based Chua's hyperchaotic system with plane of equilibria has been studied.

There are many categorization of dynamical systems based on different properties. One of them categorizes dynamical systems into two groups, systems with self-excited or systems with hidden attractors [36,37]. This categorization is based on the basin of attractions of attractors. A self-excited attractor can be found using initial conditions around an unstable equilibrium while hidden attractors cannot [38,39]. A time varying controller for the synchronization of hidden chaotic attractors has been proposed in [40].

Memristive chaotic and hyperchaotic systems have attracted lot of attentions $[41,42]$. Many studies in the context of nonlinear dynamic have been done in this area [43-48]. Chaotic dynamic of a memristive Wien-bridge oscillator has been studied in [49]. Also, fractional order chaotic systems have been a hot topic [50]. Although there are some chaotic circuits recently published, only a few focused on dynamical behaviors of the systems with fractional orders [51-53]. Motivated by this, we investigate a chaotic circuit with a first order memristor. In Section 2, the fractional order model of memristive diode bridge oscillator is introduced. In Section 3, we discuss the dynamics and multistability properties of the model. Conclusions and discussion are given in the last section.

\section{Fractional order memristive diode bridge oscillator (FOMDBO)}

Memristive based chaotic oscillators have been discussed widely in literature. We have derived the proposed memristive diode bridge oscillator (MDBO) circuit by modifying the jerk system proposed in [54]. We have replaced the two diode element with a memristor whose equivalent circuit is derived using a four diode bridge. The electronic circuit of the proposed system is presented in Figure 1. The circuit has only one nonlinear element. That element is in the form of the memristor if we assume 


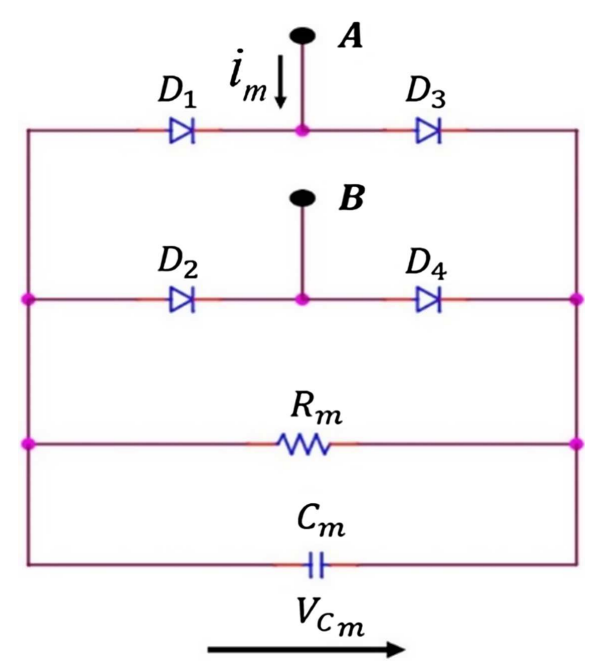

Fig. 2. Diode bridge equivalent circuit of the memristor with a first order RC filter.

that all the operational amplifiers operate in the linear region (ideal) and has three integrators and two inverting amplifiers.

To derive a new mathematical model of the circuit shown in Figure 1, we use the generalized memristor which consist of a first order filter with a diode bridge [55] as shown in Figure 2. All four diodes are chosen to be identical and the values of $R_{m}$ and $C_{m}$ are chosen such that the system shows chaotic oscillations.

The mathematical model of the current and voltage are given by,

$$
\begin{aligned}
i_{m} & =2 I_{s} e^{-\alpha V_{C_{m}}} \sinh \left(\alpha V_{C_{m}}\right) \\
\frac{d V_{C_{m}}}{d t} & =\frac{2 I_{s} R\left(e^{-\alpha V_{C_{m}}} \cosh \left(\alpha V_{C_{m}}\right)-1\right)-V_{C_{m}}}{R C_{m}}
\end{aligned}
$$

where $\alpha=\frac{1}{2 \pi \eta V_{T}}$ and $\eta, V_{T}$ are the emission coefficient and thermal voltage respectively [56]. To derive the state equations of the circuit, let us define the voltages across the capacitors $C_{a}, C_{b}, C_{c}, C_{m}$ as $V_{C_{a}}, V_{C_{b}}, V_{C_{c}}, V_{C_{m}}$ respectively. Applying Kirchoffs voltage and current laws to the circuit, the four state equations can be derived as,

$$
\begin{aligned}
C_{a} \frac{d V_{C_{a}}}{d t} & =\frac{V_{C_{b}}}{R_{b}} \\
C_{b} \frac{d V_{C_{b}}}{d t} & =\frac{V_{C_{c}}}{R_{b}} \\
C_{c} \frac{d V_{C_{c}}}{d t} & =\frac{V_{C_{a}}}{R}-\frac{V_{C_{b}}}{R_{a}}-\frac{V_{C_{c}}}{R}-2 I_{s} e^{-\alpha V_{C_{m}}} \sinh \left(\alpha V_{C_{m}}\right) \\
C_{m} \frac{d V_{C_{m}}}{d t} & =\frac{2 I_{s} R\left(e^{-\alpha V_{C_{m}}} \cosh \left(\alpha V_{C_{m}}\right)-1\right)-V_{C_{m}}}{R} .
\end{aligned}
$$

To derive the dimensionless state equations of (2), we assign the state variables as $x=V_{C_{a}}, y=V_{C_{b}}, z=V_{C_{c}}, w=V_{C_{m}}$. The parameters are taken as $a_{1}=\frac{1}{R C_{a}}$, $a_{2}=\frac{1}{R C_{b}}, a_{3}=\frac{1}{R C_{c}}, a_{4}=-\frac{1}{R C_{c}}, a_{5}=-\frac{1}{R_{a} C_{c}}, a_{6}=-\frac{2 I_{s}}{C_{m}}, a_{7}=\alpha, a_{8}=\frac{1}{C_{m}}$, 



Fig. 3. Four projections of the strange attractor of the MDBO system.

$a_{9}=-\frac{1}{R_{m} C_{m}}$. Applying these assumptions, the dimensionless state equation of the MDBO system can be derived as,

$$
\begin{aligned}
\dot{x} & =a_{1} y \\
\dot{y} & =a_{2} z \\
\dot{z} & =a_{3} x+a_{4} z=a_{5} y+a_{6} e^{-a_{7} w} \sinh \left(\frac{a_{7} x}{3}\right) \\
\dot{w} & =a_{8}\left(\left(-a_{6}\right)\left(e^{-a_{7} w} \cosh \left(\frac{a_{7} x}{3}\right)-1\right)\right)+a_{9} w .
\end{aligned}
$$

The MDBO system shows strange attractor in parameter values $a_{1}=1, a_{2}=2, a_{3}=$ $1, a_{4}=-1, a_{5}=-2.95, a_{6}=-5.36 e-5, a_{7}=5, a_{8}=0.02, a_{9}=-3$ and initial conditions $[0.1,0.1,0.1,0.1]$. Figure 3 presents the strange attractor of the MDBO system.

The equilibria of the MDBO system can be calculated as follows:

$$
\begin{aligned}
& w=-\frac{1}{a_{7}} \ln \left(\frac{-a_{3} x}{a_{6} \sinh \left(\frac{a_{7} x}{3}\right)}\right) \\
& 0=a_{8}\left(\left(-a_{6}\right)\left(e^{-a_{7} w} \cosh \left(\frac{a_{7} x}{3}\right)-1\right)\right)+a_{9} w .
\end{aligned}
$$

Equation (4) is a transcendental equation and cannot be solved analytically and hence we choose numerical solutions using MATLAB to find the equilibrium points. It can be seen that the MDBO system has a trivial fixed point $E_{1}$ at $[0,0,0,0]$ and have two nontrivial equilibrium points $E_{2}, E_{3}$ at $\mp 7.69,0,0,0.0512$. The eigenvalues of the MDBO system at the trivial equilibrium point $E_{1}$ are $\lambda_{1}=0.3166, \lambda_{2,3}=$ 
$-0.6583 \pm 2.4256 i, \lambda_{4}=-3$ and at nontrivial points $E_{2}, E_{3}$ are $\lambda_{1,2}=0.5903 \pm$ $2.9709 i, \lambda_{3}=-4.1245, \lambda_{4}=-1.8203$. As can be observed from the eigenvalues the equilibrium point $E_{1}$ is a saddle focus of index- 1 and equilibrium points $E_{2}, E_{3}$ are saddle focus of index-2.

In this paper, the fractional order memristive diode bridge oscillator (FOMDBO) has been proposed. The fractional order mathematical model of the proposed system is derived using Grünwald-Letnikov (GL). Let us define the FOMDBO oscillator as,

$$
\begin{aligned}
D^{q} x & =a_{1} y \\
D^{q} y & =a_{2} z \\
D^{q} z & =a_{3} x+a_{4} z+a_{5} y+a_{6} e^{-a_{7} w} \sinh \left(\frac{a_{7} x}{3}\right) \\
D^{q} w & =a_{8}\left(\left(-a_{6}\right)\left(e^{-a_{7} w} \cosh \left(\frac{a_{7} x}{3}\right)-1\right)\right)+a_{9} w .
\end{aligned}
$$

The discrete form of the FOMDBO system is given by,

$$
\begin{aligned}
x\left(t_{k}\right)= & \left(a_{1} y\left(t_{k-1}\right)\right) h^{q}-\sum_{j=0}^{N} \beta_{j}^{q} x\left(t_{k-j}\right) \\
y\left(t_{k}\right)= & \left(a_{2} z\left(t_{k-1}\right)\right) h^{q}-\sum_{j=0}^{N} \beta_{j}^{q} y\left(t_{k-j}\right) \\
z\left(t_{k}\right)= & \left(a_{3} x\left(t_{k-1}\right)+a_{4} z\left(t_{k-1}\right)+a_{5} y\left(t_{k-1}\right)\right. \\
& \left.+a_{6} e^{-a_{7} w\left(t_{k-1}\right)} \sinh \left(\frac{a_{7} x\left(t_{k-1}\right)}{3}\right)\right) h^{q}-\sum_{j=0}^{N} \beta_{j}^{q} z\left(t_{k-j}\right) \\
w\left(t_{k}\right)= & \left(a_{8}\left(\left(-a_{6}\right)\left(e^{-a_{7} w\left(t_{k-1}\right)} \cosh \left(\frac{a_{7} x\left(t_{k-1}\right)}{3}\right)-1\right)\right)\right. \\
& \left.+a_{9} w\left(t_{k-1}\right)\right) h^{q}-\sum_{j=0}^{N} \beta_{j}^{q} w\left(t_{k-j}\right)
\end{aligned}
$$

where $h$ is the step size, $q$ is the fractional order of the differential equation, and $\beta_{j}$ is binomial coefficients required for the numerical simulation. The value of $N$ is taken as the truncation window size $L$ and as $k$ when all the available memory elements are used.

The parameter values are considered as $a_{1}=1, a_{2}=2, a_{3}=-1, a_{4}=-1, a_{6}=$ $-5.36 e-5, a_{7}=5, a_{8}=0.02, a_{9}=-3$ and initial conditions are [0.1,0.1,0.1,0.1]. Figures 4 and 5 show the chaotic attractor of the FOMDBO system in $a_{5}=-2.95$ and $a_{5}=-1.9$. For both cases the commensurate fractional orders are kept as $q=0.99$.

\section{Dynamical properties of FOMDBO}

\subsection{Route to chaos}

To discuss the dynamical properties of FOMDBO, bifurcations with respect to changing parameters and fractional order of the system is investigated. The bifurcation parameter has been chosen as $a_{2}$ while the other parameters are kept constant as 

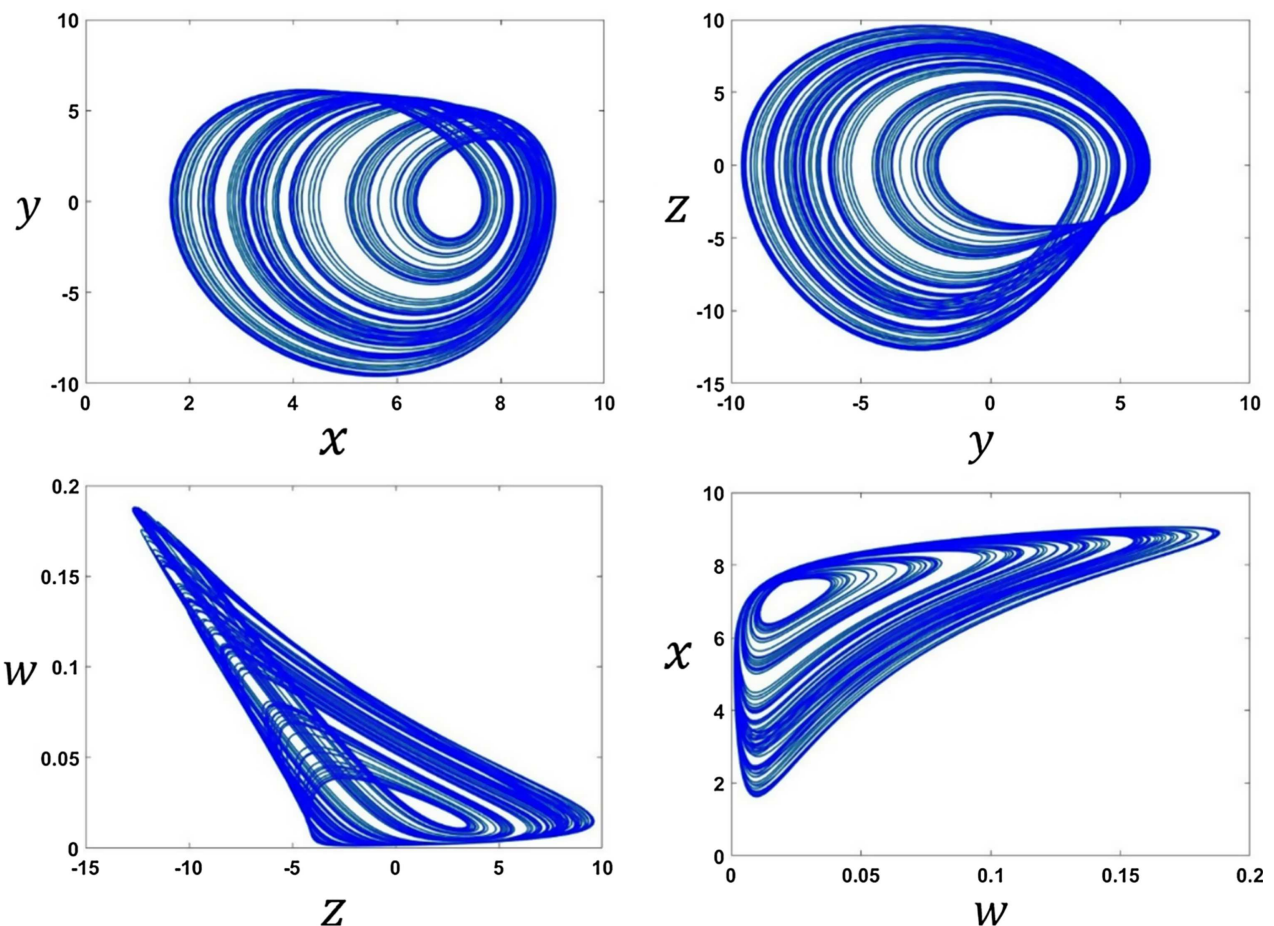

Fig. 4. 2D phase portraits of the FOMDBO in $a_{5}=-2.95$.
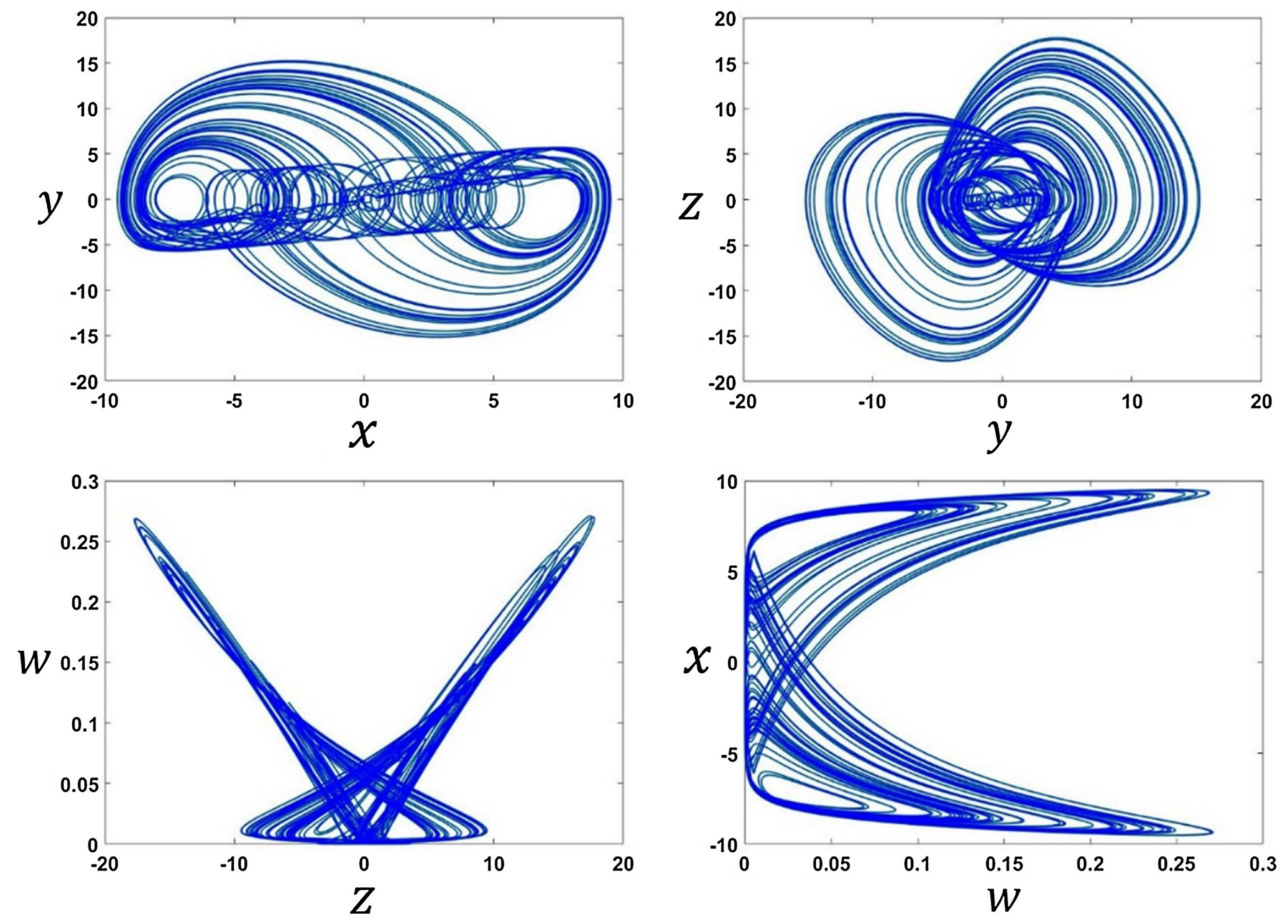

Fig. 5. 2D phase portraits of the FOMDBO system in $a_{5}=-1.9$. 

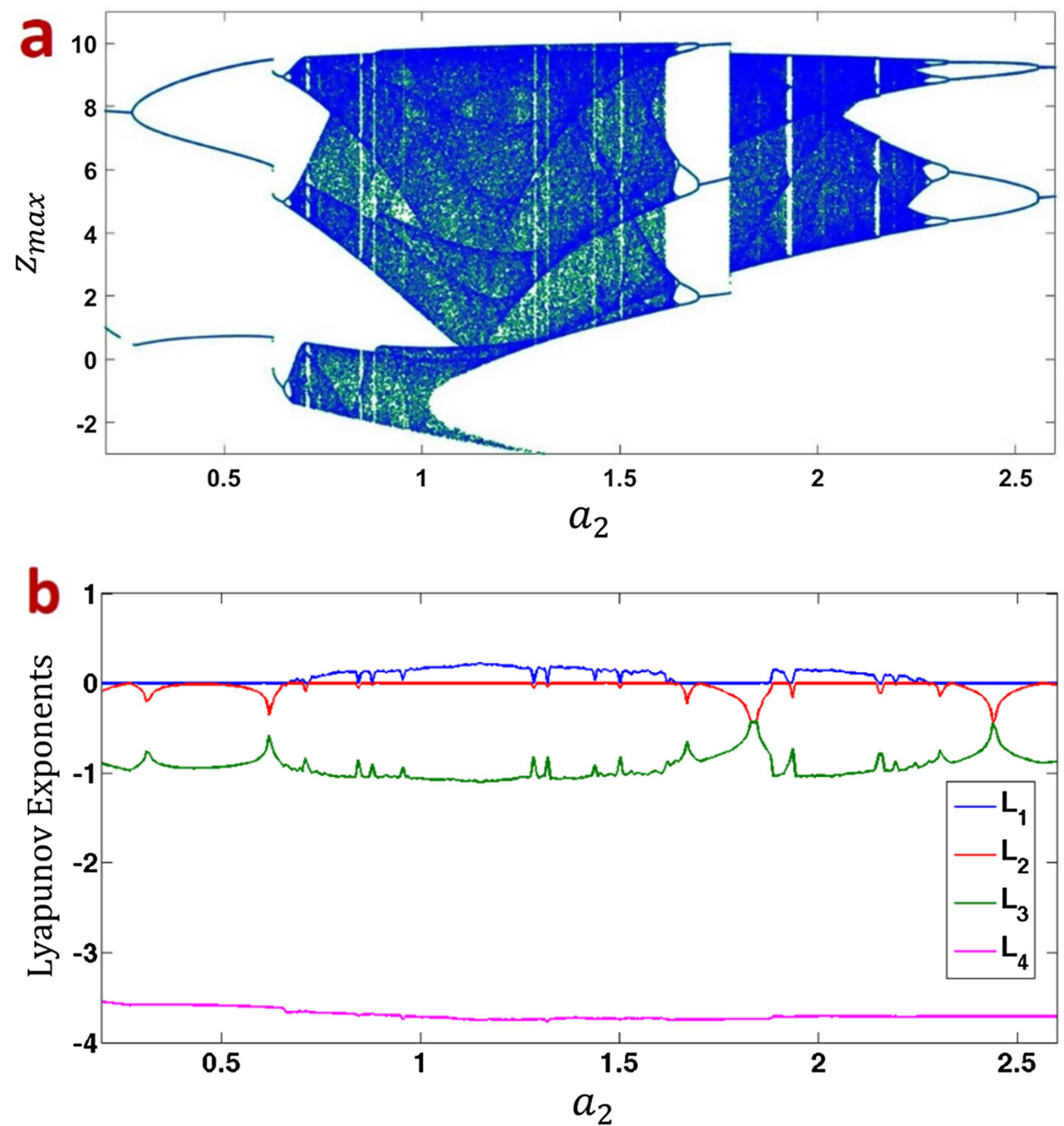

Fig. 6. (a) Bifurcation of the FOMDBO system with respect to changing parameter $a_{2}$; and (b) the corresponding Lyapunov exponents.

$a_{1}=1, a_{3}=-1, a_{4}=-1, a_{5}=-2.95, a_{6}=-5.36 e-5, a_{7}=5, a_{8}=0.02$, $a_{9}=-3$ and the commensurate fractional order of the system is taken $q=0.995$. Initial conditions are considered $[0.1,0.1,0.1,0.1]$. Bifurcation diagram of FOMDBO and Lyapunov exponents (LEs) with respect to changing parameter $a_{2}$ are shown in Figure 6 . The FOMDBO shows a period doubling route to chaos and has creation and annihilation of period doublings which is known as antimonotonicity. This property of the system will be investigated in detail at a later part of this paper.

Next, we investigate bifurcation diagram of FOMDBO with respect to changing commensurate fractional order. In this case, we fix all the other system parameters except $a_{5}$ to their respective values (chaotic solution) and initial conditions taken as $[0.1,0.1,0.1,0.1]$. The bifurcation is derived for two conditions, $a_{5}=-2.95$ and $a_{5},=-1.9$ and they are shown in Figures $7 \mathrm{a}$ and $7 \mathrm{~b}$ respectively. In $a_{5}=-2.95$, the system shows chaotic oscillations in the interval $0.987 \leq q<1$ which can be seen in Figures $7 \mathrm{a}$ and $7 \mathrm{~b}$ show antimonotonicity by period doubling in $0.975 \leq q<0.98$ and reverse route of period doubling in $0.992 \leq q<0.995$. 

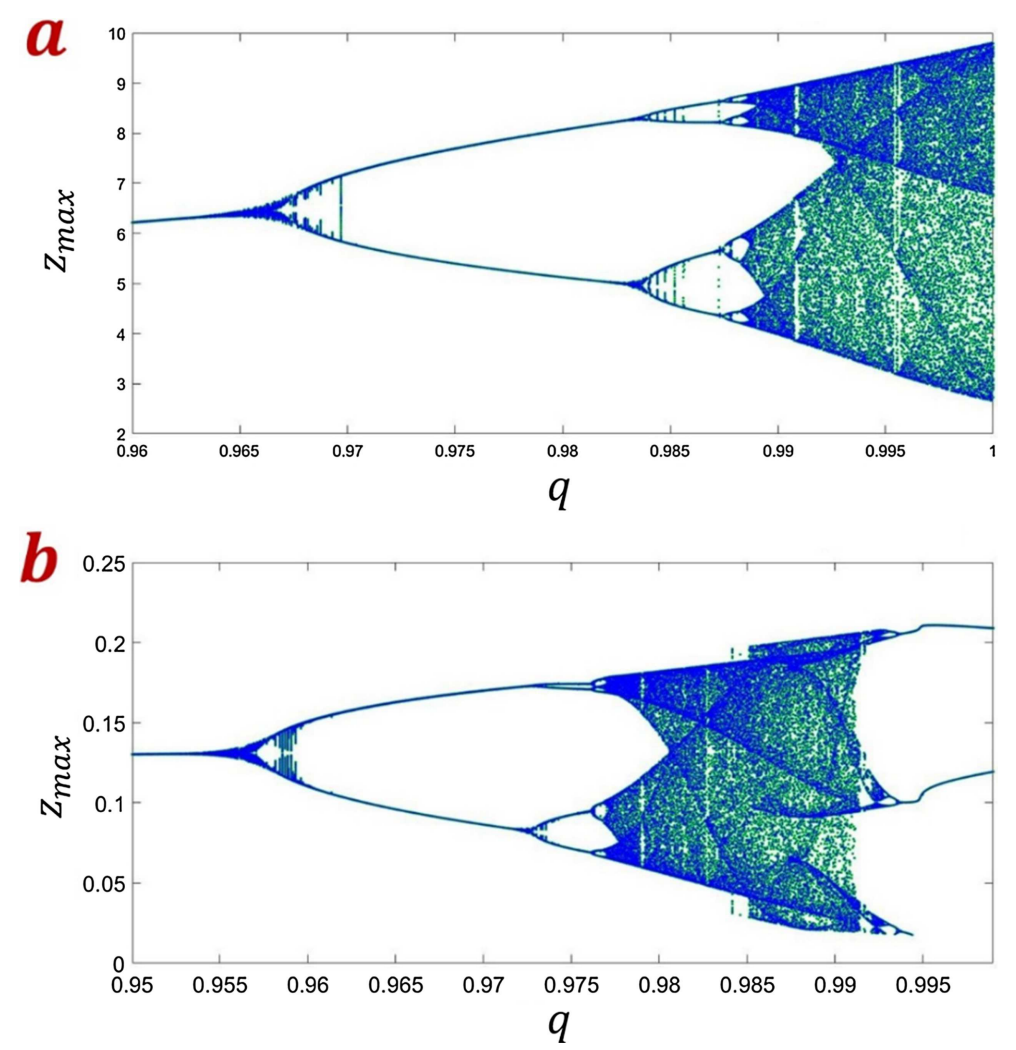

Fig. 7. (a) Bifurcation of the FOMDBO system with respect to changing fractional order $q$ in $a_{5}=-2.95$. (b) Bifurcation of the FOMDBO system with respect to changing fractional order $q$ in $a_{5}=-1.9$.

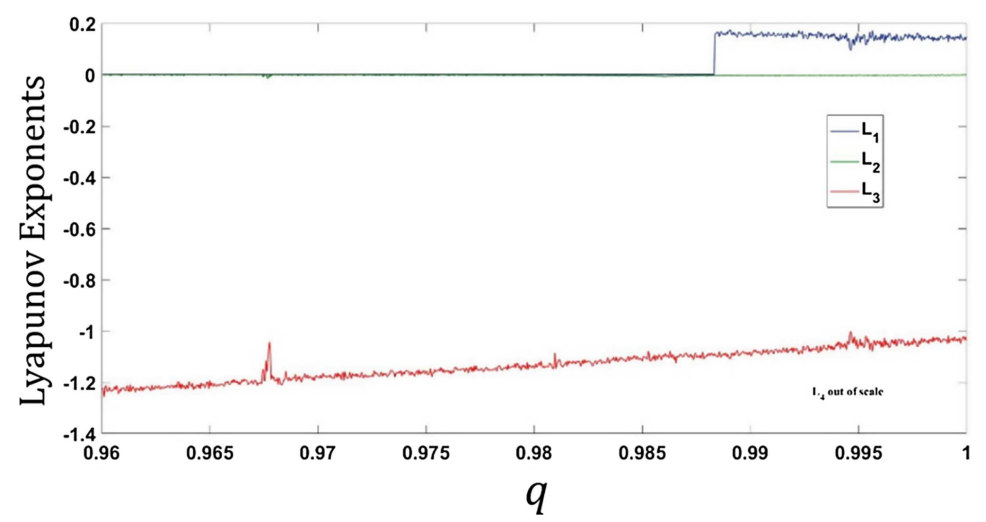

Fig. 8. Lyapunov spectrum of the FOMDBO system in $a_{5}=-2.95$ with respect to changing fractional order $q$.

The Lyapunov exponents (LEs) of the FOMDBO in $a_{5}=-2.95$ are shown in Figure 8. Lyapunov exponents are derived using the Wolf's algorithm [57] and the fractional order predictor-corrector [58] solver fde12 [59] instead of the ode solvers [60]. The positive largest Lyapunov exponent proves the existence of chaos in higher values of fractional order $q$. 



Fig. 9. (a) Bifurcation of the FOMDBO with respect to changing parameter $a_{2}$ with forward (blue plot) and backward (red plot) continuation, (b) the corresponding Maximum Lyapunov exponent (MLE) with forward (blue plot) and backward (red plot) continuation.

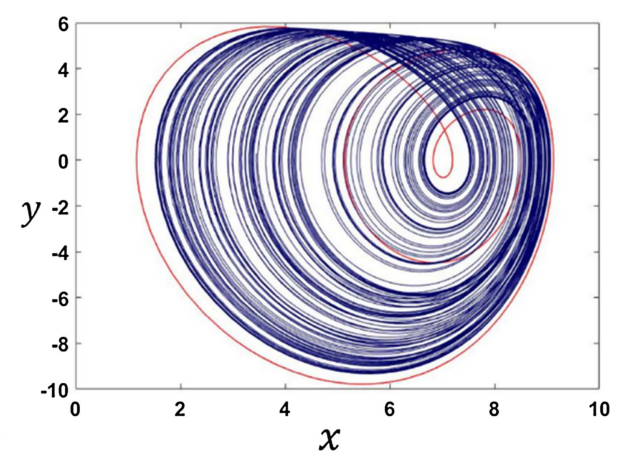

Fig. 10. Coexisting attractors of FOMDBO in $a_{2}=1.82$. Initial conditions are $[7.03,4.77,1.05,0]$ in blue plot and $[6.4,-6.5,-0.59,0.06]$ in red plot.

\subsection{Multistability analysis}

In order to investigate multistability of a system, we should track the attractors of the system with different initial conditions. One common way to detect multistability in a dynamical system is plotting bifurcation diagram of the system with forward and backward continuation. Parameter $a_{5}=-2.95$, commensurate fractional order taken 


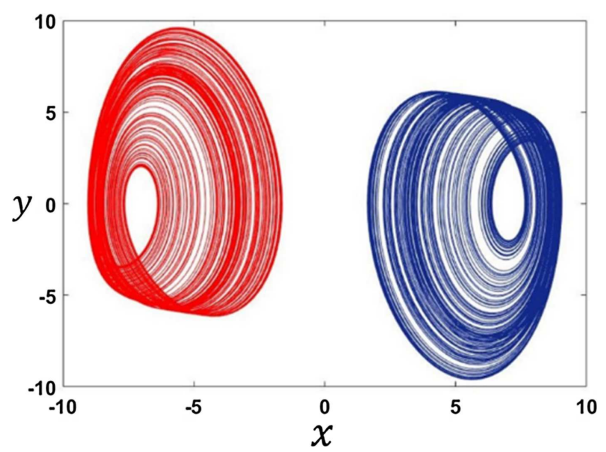

Fig. 11. Coexisting attractors of FOMDBO in $a_{2}=2$. Initial conditions are $[0.1,0.1,0.1,0.1]$ in blue plot and $[-0.1,-0.1,-0.1,-0.1]$ in red plot.

as $q=0.99$ and the other parameters are fixed to their respective chaotic solutions. In the forward (or backward) continuation method bifurcation diagram is obtained by plotting local maxima of the coordinate $z$ in terms of the parameter that is increased (or decreased) in tiny steps in the range of $0.5 \leq a_{2} \leq 3$. The final states at each parameter serves as the initial conditions for the next parameter. Plotting bifurcation diagram with forward and backward continuation represents a simple way to localize the window in which the system develops multistability. In Figure 9, the existence of a chaotic attractor in forward continuation in the interval $1.784 \leq a_{2} \leq 1.887$ can be seen while the backward continuation shows period 3 limit cycles in that range. Also, a very narrow band of chaotic attractor can be seen in $2.155 \leq a_{2} \leq 2.163$ with forward continuation. But in the backward continuation a period 6 limit cycle can be seen. These claims are well supported by the finite time Lyapunov exponents (only the maximum LE is plotted) shown in Figure 9b. They are calculated using the Wolf's algorithm [57,60-62] with re-initialized initial conditions obtained in forward and backward continuations. So, the proposed FOMDBO system has hysteresis since the bifurcation diagram with forward and backward continuation are different in some interval of parameters. In other words, the system shows bi-stability. Figure 10 shows the coexisting attractor of FOMDBO in $a_{2}=1.82$ and two different initial conditions. The FOMDBO shows a chaotic attractor with coexisting limit cycles as shown with blue and red plots respectively. Figure 11 presents coexisting chaotic attractors in $a_{2}=2$ with two antisymmetric initial conditions.

\subsection{Antimonotonicity}

The process of period doubling and its inverse route which can occur in the bifurcation diagram of a system is termed as antimonotonicity. To investigate this in detail, we derive the bifurcation of FOMDBO with $a_{2}$ and $a_{5}$ as the control parameters. The fractional order in this analysis is taken as $q=0.99$ and we use the forward continuation to plot the bifurcation diagrams. Figures $12 \mathrm{a}-\mathrm{f}$ show the bifurcation diagrams of the FOMDBO with respect to changing parameter $a_{2}$ and in various values of control parameter $a_{5}$. The novelty of this study is that such a feature has not been discussed in literatures with fractional order models. The evidence of period doubling route to chaos and its inverse route is clearly seen from the bifurcation plots reported in Figure 12.

\section{Conclusions}

A fractional order memristor diode bridge oscillator was proposed and dynamical properties of the proposed oscillator have been discussed. The fractional order model 

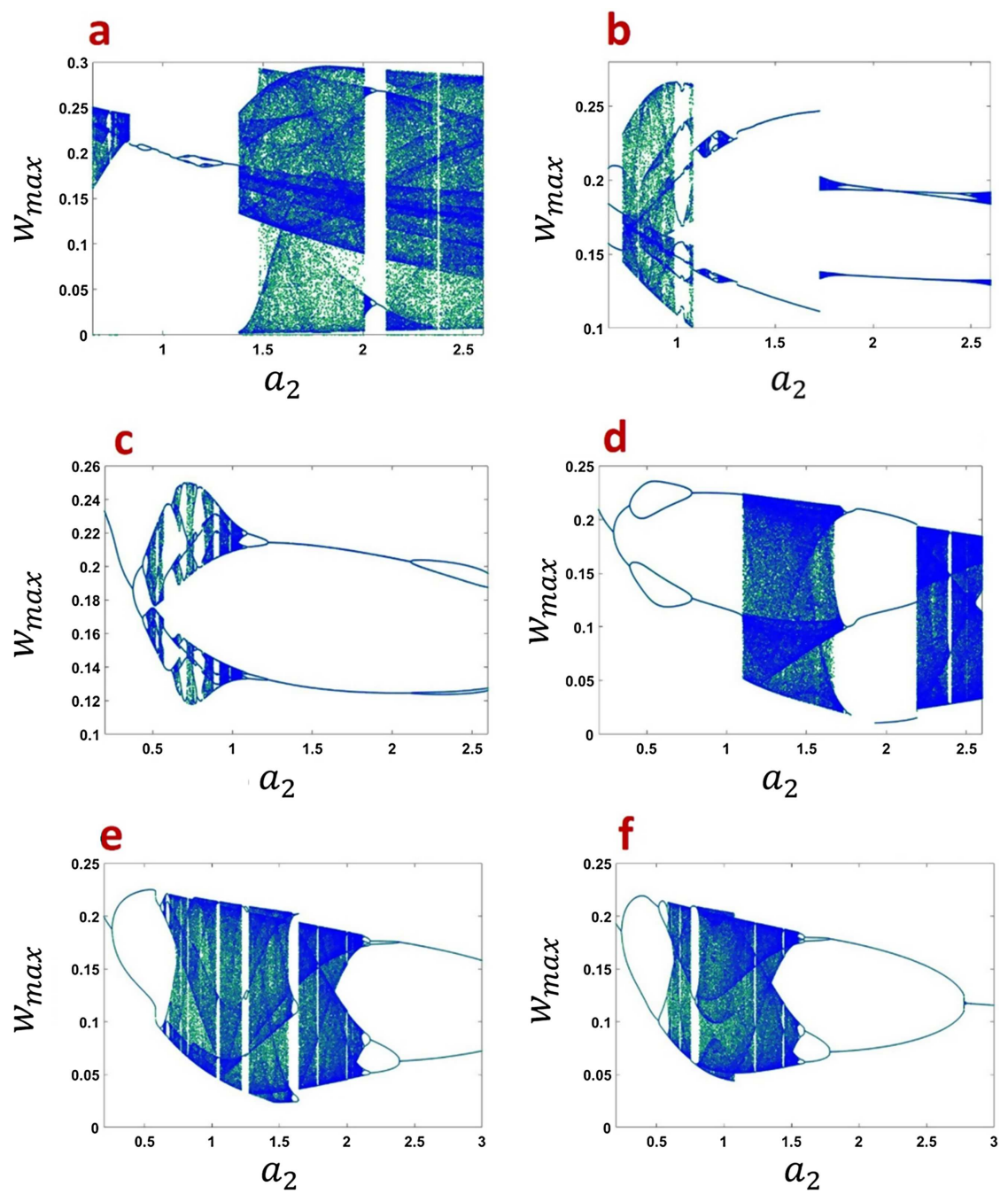

Fig. 12. Bifurcation diagram of FOMDBO with respect to changing $a_{2}$ and for different constant values of $a_{5}$. (a) $a_{5}=-1.7$, (b) $a_{5}=-2.2$, (c) $a_{5}=-2.5$, (d) $a_{5}=-2.8$, (e) $a_{5}=-3$, (f) $a_{5}=-3.2$.

was derived using Grünwald-Letnikov. FOMDBO system showed multistability and coexisting attractors which were investigated in detail. In addition, the fractional order system exhibited antimonotonicity property which was investigated by keeping two control parameters and deriving the bifurcation plots. As far as we know such properties were not discussed in the literature with fractional order models.

This work was partially supported by the National Natural Science Foundation of China (61503133), the Open Project of MOE Key Laboratory of Image Processing and Intelligence Control (IPIC2015-04), and the Natural Science Foundation of Hunan Province (2016JJ6043). The work was supported by the College of Engineering, Defence University, Ethiopia with partial support from the Center for Nonlinear Dynamics, Defence University, Ethiopia (Grant No.- CND/DEC/2018/1) and partially supported by Iran Science Elites Federation Grant No. M - 97171. 


\section{References}

1. J.C. Sprott, Phys. Rev. E 50, R647 (1994)

2. S. Jafari, J.C. Sprott, S.M.R. Hashemi Golpayegani, Phys. Lett. A 377, 699 (2013)

3. S. Jafari, J.C. Sprott, Chaos Solitons Fractals 57, 79 (2013)

4. M. Molaie, S. Jafari, J.C. Sprott, S.M.R.H. Golpayegani, Int. J. Bifurc. Chaos 23, 1350188 (2013)

5. Z. Wei, R. Wang, A. Liu, Math. Comput. Simul. 100, 13 (2014)

6. V.T. Pham, S. Jafari, C. Volos, X. Wang, S.M.R.H. Golpayegani, Int. J. Bifurc. Chaos 24, $1450146(2014)$

7. F. Nazarimehr, K. Rajagopal, J. Kengne, S. Jafari, V.T. Pham, Chaos Solitons Fractals 111, 108 (2018)

8. S. Ren, S. Panahi, K. Rajagopal, A. Akgul, V.T. Pham, S. Jafari, Z. Naturforsch. A 73, 239 (2018)

9. Z. Wei, I. Moroz, A. Liu, Turk. J. Math. 38, 672 (2014)

10. V.T. Pham, S. Jafari, T. Kapitaniak, C. Volos, S.T. Kingni, Int. J. Bifurc. Chaos 27, $1750053(2017)$

11. V.T. Pham, X. Wang, S. Jafari, C. Volos, T. Kapitaniak, Int. J. Bifurc. Chaos 27, $1750097(2017)$

12. X. Wang, V.T. Pham, S. Jafari, C. Volos, J.M. Munoz-Pacheco, E. Tlelo-Cuautle, IEEE Access 5, 8851 (2017)

13. J.P. Singh, B. Roy, Nonlinear Dyn. 89, 1 (2017)

14. V.T. Pham, C. Volos, T. Kapitaniak, S. Jafari, X. Wang, Int. J. Electron. 105, 385 (2018)

15. S. Panahi, Z. Aram, S. Jafari, V.T. Pham, C. Volos, K. Rajagopal, Pramana 90, 31 (2018)

16. J.P. Singh, B.K. Roy, S. Jafari, Chaos Solitons Fractals 106, 243 (2018)

17. S. Jafari, J.C. Sprott, V.T. Pham, C. Volos, C. Li, Nonlinear Dyn. 86, 1349 (2016)

18. S. Jafari, J.C. Sprott, M. Molaie, Int. J. Bifurc. Chaos 26, 1650098 (2016)

19. K. Rajagopal, S. Jafari, A. Karthikeyan, A. Srinivasan, B. Ayele, Circuits Syst. Signal Process. 37, 1 (2018)

20. K. Rajagopal, S. Çiçek, A.J.M. Khalaf, V.T. Pham, S. Jafari, A. Karthikeyan, P. Duraisamy, Z. Naturforsch. A 73, 609 (2018)

21. E. Tlelo-Cuautle, L.G. De La Fraga, V.T. Pham, C. Volos, S. Jafari, A. de Jesus QuintasValles, Nonlinear Dyn. 89, 1 (2017)

22. C. Li, K. Su, L. Wu, J. Comput. Nonlinear Dyn. 8, 031005 (2013)

23. C. Li, Y. Tong, Pramana 80, 583 (2013)

24. Y. Zhang, Phys. Lett. A 377, 1269 (2013)

25. J. Ma, P. Zhou, B. Ahmad, G. Ren, C. Wang, PLoS One 13, e0191120 (2018)

26. W. Ai, K. Sun, Y. Fu, Int. J. Mod. Phys. C 29, 1850049 (2018)

27. J.C. Sprott, S. Jafari, A.J.M. Khalaf, T. Kapitaniak, Eur. Phys. J. Special Topics 226, $1979(2017)$

28. Y.-X. Tang, A.J.M. Khalaf, K. Rajagopal, V.-T. Pham, S. Jafari, Y. Tian, Chin. Phys. B 27, $40502(2018)$

29. C. Li, J.C. Sprott, Y. Mei, Nonlinear Dyn. 89, 2629 (2017)

30. Z. Wei, V.T. Pham, A.J.M. Khalaf, J. Kengne, S. Jafari, Int. J. Bifurc. Chaos 28, 1850085 (2018)

31. B. Bao, T. Jiang, G. Wang, P. Jin, H. Bao, M. Chen, Nonlinear Dyn. 89, 1157 (2017)

32. B. Bao, T. Jiang, Q. Xu, M. Chen, H. Wu, Y. Hu, Nonlinear Dyn. 86, 1711 (2016)

33. B.C. Bao, H. Bao, N. Wang, M. Chen, Q. Xu, Chaos Solitons Fractals 94, 102 (2017)

34. H. Bao, N. Wang, B. Bao, M. Chen, P. Jin, G. Wang, Commun. Nonlinear Sci. Numer. Simul. 57, 264 (2018)

35. J.P. Singh, B. Roy, Optik 145, 209 (2017)

36. N.V. Stankevich, N.V. Kuznetsov, G.A. Leonov, L.O. Chua, Int. J. Bifurc. Chaos 27, 1730038 (2017)

37. N.V. Kuznetsov, G.A. Leonov, M.V. Yuldashev, R.V. Yuldashev, Commun. Nonlinear Sci. Numer. Simul. 51, 39 (2017) 
38. P.R. Sharma, M.D. Shrimali, A. Prasad, N.V. Kuznetsov, G.A. Leonov, Eur. Phys. J. Special Topics 224, 1485 (2015)

39. M.-F. Danca, N. Kuznetsov, Chaos Solitons Fractals 103, 144 (2017)

40. J.P. Singh, B.K. Roy, Trans. Inst. Meas. Control 40, 3573 (2017)

41. B. Bao, A. Hu, H. Bao, Q. Xu, M. Chen, H. Wu, Complexity 2018, 3872573 (2018)

42. Y. Zhang, L.F. Lu, Nonlinear Dyn. 77, 1121 (2014)

43. B. Bao, L. Xu, Z. Wu, M. Chen, H. Wu, Int. J. Electron., 105, 1159 (2018)

44. Y. Zhang, Nonlinear Dyn. 73, 2221 (2013)

45. B.C. Bao, P.Y. Wu, H. Bao, H.G. Wu, X. Zhang, M. Chen, Chaos Solitons Fractals 109, 146 (2018)

46. B.C. Bao, P.Y. Wu, H. Bao, Q. Xu, M. Chen, Chaos Solitons Fractals 106, 161 (2018)

47. H. Bao, N. Wang, H. Wu, Z. Song, B. Bao, IETE Tech. Rev. 36, 1 (2019)

48. Q. Xu, Q.L. Zhang, H. Qian, H.G. Wu, B.C. Bao, Int. J. Circuit Theory Appl. 46, 1917 (2018)

49. H. Wu, B. Bao, Z. Liu, Q. Xu, P. Jiang, Nonlinear Dyn. 83, 893 (2016)

50. Y. Zhang, G. Luo, Q. Cao, M. Lin, Int. J. Nonlinear Mech. 58, 151 (2014)

51. X. Wang, S.T. Kingni, C. Volos, V.T. Pham, D. Vo Hoang, S. Jafari, Int. J. Electron. 106, 109 (2019)

52. K. Rajagopal, F. Nazarimehr, A. Karthikeyan, A. Srinivasan, S. Jafari, Asian J. Control 201979 (2018)

53. K. Rajagopal, A. Akgul, S. Jafari, B. Aricioglu, Nonlinear Dyn. 91, 957 (2018)

54. Z. Njitacke, L.K. Kengne, Chaos Solitons Fractals 105, 77 (2017)

55. B. Bao, J. Yu, F. Hu, Z. Liu, Int. J. Bifurc. Chaos 24, 1450143 (2014)

56. M.P. Hanias, G. Giannaris, A. Spyridakis, A. Rigas, Chaos Solitons Fractals 27, 569 (2006)

57. A. Wolf, J.B. Swift, H.L. Swinney, J.A. Vastano, Physica D 16, 285 (1985)

58. K. Diethelm, A.D. Freed, Forschung und wissenschaftliches Rechnen 1999, 57 (1998)

59. R. Garrappa, Predictor-corrector PECE method for fractional differential equations. MATLAB Central File Exchange [File ID: 32918] (2011)

60. M.-F. Danca, Nonlinear Dyn. 81, 227 (2015)

61. I. Petráš, Fractional-order nonlinear systems, nonlinear physical science (Higher Education Press and Springer, Beijing, 2011)

62. M.F. Tolba, A.M. AbdelAty, N.S. Soliman, L.A. Said, A.H. Madian, A.T. Azar, A.G. Radwan, AEU Int. J. Electron. Commun. 78, 162 (2017) 